\title{
Social Disinhibition: Piloting a New Clinical Measure in Individuals with Traumatic Brain Injury
}

\author{
Cynthia A. Honan, ${ }^{1,5}$ Samantha K. Allen, ${ }^{2}$ Alana Fisher, ${ }^{3}$ Katherine Osborne-Crowley, ${ }^{4}$ \\ and Skye McDonald ${ }^{4,5}$ \\ ${ }^{1}$ Department of Psychology, School of Medicine, University of Tasmania, Launceston, Tasmania, Australia \\ ${ }^{2}$ School of Psychology, University of New South Wales, Sydney, Australia \\ ${ }^{3}$ School of Psychology, Centre for Medical Psychology and Evidence-Based Decision Making (CeMPED), \\ University of Sydney, Sydney, Australia \\ ${ }^{4}$ School of Psychology, University of New South Wales, Sydney, Australia \\ ${ }^{5}$ Moving Ahead NHMRC Centre for Research Excellence in Brain Recovery, Sydney, Australia
}

\begin{abstract}
Social disinhibition difficulties are common following traumatic brain injury (TBI). However, clinically sensitive tools to objectively assess the difficulties are lacking. This study aimed to pilot a new clinical measure of social disinhibition, the social disinhibition task (SDT). Whether social disinhibition is dependent on the type of social information judgements required and whether disinhibited responses can be adjusted with additional guidance were also examined. Participants were 31 adults (25 Male) with moderate-to-severe TBI and 22 adult (17 Male) healthy control participants. Participants viewed scenes of complex social situations and were asked to describe a character in them (Part A), describe a character while inhibiting inappropriate or negative responses (Part B), and describe a character while not only inhibiting negative responses, but also providing positive responses (Part C). One-half of the items contained a faux pas requiring participants to make inferences about a character's mental state. TBI and control participants responded similarly to Part A, although control participants responded less positively than TBI participants in the faux pas items. TBI participants were significantly impaired on Part $\mathrm{B}$ indicating they experienced difficulties in inhibiting automatic responding. TBI participants were however able to adjust their responding in Part $\mathrm{C}$ so that they respond similarly to the control participants. Between group differences were not detected in reaction time. Overall, the SDT appears to be suitable to detect social inhibition difficulties in clinical settings and provides a new direction for remediation of the difficulties in individuals with TBI.
\end{abstract}

Keywords: social cognition, traumatic brain injury, social disinhibition, theory of mind

Poor psychosocial functioning is common after severe traumatic brain injury (TBI). It is detrimental to family and social relations and to gaining and maintaining employment (Tate, Broe, Cameron, Hodgkinson, \& Soo, 2005). A potential source of such problems is difficulties in social inhibition or a person's ability to self-regulate behaviour and emotion in social settings (Osborne-
Crowley, McDonald, \& Francis, 2015). Often attributed to compromised activity in the frontal (particularly the orbitofrontal and lateral prefrontal cortex), insular, and temporal lobe regions of the brain (Hooker \& Knight, 2006; Knutson et al., 2015; Lee et al., 2010; Løvstad et al., 2012), these difficulties manifest as the production of socially inappropriate verbal, physical or sexual acts

Address for correspondence: Dr Cynthia A Honan, Department of Psychology, School of Medicine, University of Tasmania, Launceston, Tasmania.E-mail: cynthia.honan@utas.edu.au 
and are said to be a reflection of poor inhibition abilities or an inability to conform to social expectations and behavioural norms (Arciniegas \& Wortzel, 2014).

Social disinhibition difficulties in individuals with TBI have been consistently reported in the literature. Many of these studies have used selfand informant-report measures to indicate the extent of social disinhibition difficulties (e.g., Barrett, McLellan, \& McKinlay, 2013; Ciurli, Formisano, Bivona, Cantagallo, \& Angelelli, 2011; Kilmer et al., 2006; Monsalve, Guitart, Lopez, Vilasar, \& Quemada, 2012; O'Keeffe, Dockree, Moloney, Carton, \& Robertson, 2007). Based upon informant reporting, it has been estimated that around $30 \%$ of individuals with moderate-to-severe TBI experience social disinhibition difficulties (Ciurli et al., 2011; Monsalve et al., 2012). This proportion is consistent with prevalence estimates of other impairments within the domain of social cognition in TBI individuals (e.g., Honan, McDonald, Sufani, Hine, \& Kumfor, 2016).

Social disinhibition difficulties have also been demonstrated using researcher-rated observational measures and experimental tasks. Most recently, Osborne-Crowley et al. (2015) found that individuals with TBI were less appropriate in their disclosure of information when asked to talk in an interview-type format about themselves and their experiences. Other recent studies have employed novel tasks to examine inhibition deficits using socially relevant information. In one such study, individuals experienced difficulty in not only inhibiting automatic thoughts (about features of a holiday destination), but also inhibiting self-referential thoughts in order to cater to the perspective of another person (in this case describing features of a holiday that would be appealing for a hypothetical guest) (McDonald et al., 2014). Similar inhibition difficulties in TBI participants were found in an alternative study requiring individuals to inhibit knowledge about a particular news story, in order to produce unrelated responses (Honan, McDonald, Gowland, Fisher, \& Randall, 2015).

Despite the presence of social disinhibition difficulties following moderate-to-severe TBI and their potential detrimental impact on rehabilitation and community integration, clinically sensitive tools to objectively assess these are lacking. Similar to the research literature, much of what is postulated about disinhibited behaviours in a clinical context is inferred from self- and informant-report questionnaires or from patient interviews (McDonald, Honan, Kelly, Byom, \& Rushby, 2013). Examples of behaviours that are used to indicate a disinhibition syndrome include saying or doing things that is considered rude or embarrassing to others, saying things without first thinking about what is being said, disclosing information that is inappropriate, greeting strangers as if they were a close friend, talking out of turn, making insensitive comments, and using inappropriate language (Beer, John, Scabini, \& Knight, 2006; Malloy, Bihrle, Duffy, \& Cimino, 1993; Rolls, Hornak, Wade, \& McGrath, 1994). Although such reporting provides the clinician with qualitative information about the types of socially disinhibited behaviours the patient may engage in, such subjective reports suffer from potential bias and under- or over-reporting of disinhibited responding in real-life social situations.

Pieces of evidence for social inhibition deficits are also often inferred from performance on tests of response inhibition or inhibitory interference control. Response inhibition ability is typically measured using go/no-go tasks such as the Conners Continuous Performance Test (Conners \& Staff, 2000) that requires suppression of an activated motor response (Nigg, 2000). Performance on these tasks is measured by the number of inhibition failures or the speed of the inhibition process (stop-signal reaction time). Inhibitory interference control, on the other hand, is typically assessed in clinical practice using measures such as the Stroop task (e.g., Stroop Neuropsychological Screening Test; Trenerry, 1989) or the Hayling Sentence Completion Task (Burgess \& Shallice, 1997). These tasks require effortful inhibition in order to suppress competing automatic response in favour of making a correct response (Nigg, 2000). Performance is measured by the number of correct responses and is a reflection of the processing speed demands of the task (e.g., number of correct responses within a time limit or reaction time). One recent meta-analysis by DimoskaDi Marco, McDonald, Kelly, Tate and Johnstone (2011) found greater support for response inhibition deficits in TBI with a moderate-to-strong overall effect size reported compared to inhibitory interference control deficits (based on studies using the Stroop tasks) with an overall minimal effect size reported. Inhibitory control difficulties as measured using the Hayling Sentence completion tasks have been more consistently reported in individuals with TBI (Draper \& Ponsford, 2008; SenathiRaja, Ponsford, \& Schönberger, 2010; Wood \& Rutterford, 2006). The extent to which these existing measures of response inhibition and inhibitory interference control are predictive of social disinhibition difficulties, however, is not known. Although by definition, social inhibition ability is likely to involve a combination of motor response inhibition and inhibitory control mechanisms, these existing tests do not contain items 
imbedded in a social context. Consequently, they are unlikely to reflect the true nature of social disinhibition difficulties experienced in individuals with TBI.

Of possible relevance to the production of controlled inhibitory responses in complex social settings is the social information that an individual focuses on when making a response. Theory of mind (ToM) difficulties, a common outcome of TBI (Honan et al., 2015; McDonald \& Flanagan, 2004; McDonald et al., 2014; Milders, Ietswaart, Crawford, \& Currie, 2008; Milders, Ietswaart, Currie, \& Crawford, 2006; Muller et al., 2010), may mean important social information is not processed or properly attended to, thus potentially impeding the individual's ability to produce the desired socially appropriate response. ToM is a specific term used to describe the ability to perceive social cues, use these to make inferences about the mental states of other people, and to use these representations to not only understand, but also predict and judge the utterances and social behaviours of others (Bibby \& McDonald, 2005; Brownell \& Martino, 1988; Ochsner \& Lieberman, 2001; Premack $\&$ Woodruff, 1978). Recent research also indicates that individuals with TBI may have specific difficulties in the ability to inhibit their own selfreferential thoughts in order to understand the perspective of others (McDonald et al., 2014). Thus, it may indeed be that inhibition responses vary depending on whether ToM is required.

The primary purpose of this study was to pilot a potential new measure of social disinhibition, the social disinhibition task (SDT), that is suitable for use in clinical practice. One assumption of the SDT is that it is a valid test of disinhibition, and thus convergent validity will also be examined. The SDT was designed to assess an individual's ability to inhibit automatic responding to negative social information and to substitute positive, socially acceptable responses. This study also aimed to determine whether social disinhibition is dependent on the type of social information judgements required. More specifically, it aimed to determine whether ToM ability moderates social inhibition ability. A supplementary aim of the study was to determine whether individuals with TBI are able to adjust their responding when provided with additional guidance. Establishing whether individuals are able to adjust their inappropriate responses is an important consideration for rehabilitation of social disinhibition difficulties in individuals with TBI.

Using the newly developed SDT, it was hypothesised that individuals with TBI will display difficulties in inhibiting automatic negative responses and have differentially more problems with items requiring ToM ability relatively to demographi- cally matched control adults. It was also hypothesised that individuals with TBI would be able to adjust their responding when provided with specific guidance about the type of response required. In relation to the examination of the measure's convergent validity, it was hypothesised that total inhibition scores would correlate with another standard measure of inhibition, namely the Hayling Sentence Completion Test (Burgess \& Shallice, 1997) and that the SDT would correlate with tests of executive function including generativity and flexibility. Given the social nature of the SDT, it was further hypothesised that the SDT would correlate with a standard test of social cognition. Finally, it was hypothesised that if the SDT is an ecologically valid test of social inhibition, it should predict socially disinhibited behaviour in everyday life as reported by self and a close other.

\section{Method}

\section{Participants}

Thirty-one adults (25 Male) with moderate-tosevere TBI and 22 adult (17 Male) healthy controls participated in this pilot study conducted over an 18-month period between 2013 and 2015. Participants with TBI were recruited from the outpatient records of three metropolitan brain-injury units in Sydney. Control participants were recruited through community notices and advertisements. Groups were matched for age $(p=.389)$, education $(p=.096)$, and gender $(p=.765)$. Mean age was 45.13 years $(S D=15.56$; range $=18-69$ years), mean education was 13.45 years $(S D=2.73$; range $=9-22$ years $)$, mean posttraumatic amnesia (PTA) was 59.19 days $(S D=$ 46.64 ; range $=3-189$ days), and mean time since injury was 12.48 years $(S D=10.49$; range $=1-$ 45 years) for the TBI participants. Mean age was 48.55 years $(S D=11.72$; range $=22-68$ years $)$, and mean education was 14.59 years $(S D=1.87$; range $=11-18$ years) for the control participants. Only a subset of 23 adults (19 Male) with TBI and 12 adult ( 10 Male) healthy control participants completed Part C of the SDT (see Measures section for details). These subsets were matched for age $(p=.610)$ and gender $(p=.513)$, but not years of education $(p=.045)$.

All participants met the following inclusion criteria: they had sustained a severe TBI resulting in at least one day in a period of PTA (Russell \& Smith, 1961; Teasdale, 1995), were discharged from hospital and living in the community, and had English as their primary spoken language. The period of PTA was assessed using the Westmead PTA scale (Marosszeky, 1998) or was 
assessed retrospectively through self-reporting at interview. Participants were excluded if they had uncorrected hearing or vision loss (as determined by an eye or hearing specialist), a current diagnosed drug and/or alcohol addiction, active psychosis or psychiatric condition, dementia or other neurodegenerative disease (as defined by the Diagnostic and Statistical Manual of Mental Disorders (DSM)-IV), aphasia, agnosia, or profound amnesia (informed through self-report or prior clinical assessment). All eligible individuals who contacted the researchers or who were contacted by the researchers agreed to participate in the study.

\section{Measures}

Social Disinhibition Task (SDT). Participants viewed a series of colour photographs of complex 'awkward' social situations comprising of two to three individuals interacting while being simultaneously told a brief description about the scene (one to two brief sentences). For example, the participant was shown a photograph of a young man (Noah) who was showing (interrupting) a young lady (Mary) while she was reading a textbook in a library (this is an example of a faux pas item). At the same time, the participant was provided with the following information: 'Mary was trying to do some last minute study for her law exam. Noah wanted to tell Mary all about the novel that he just finished reading' (this was read to the participant and was written in text at the top of each photograph), 'Tell me what you think of Noah?'. In another example, the participant was shown a photograph of a noticeably angry young lady (Nadine) who was sitting on a park bench with her boyfriend (Jack) (this is an example of a non-faux pas item). At the same time, the participant was provided with the following information: 'Nadine was annoyed that her boyfriend Jack forgot her birthday', 'Tell me what you think of Jack'. There were three parts to the test, each with 10 items. Part A (control task) required participants to say the 'first word or thing' that came to mind about a particular character in the scene as quickly as possible. Part B (inhibition task) required participants to not say anything negative that might offend the person. They were also asked not to comment on the person's age, size, race, ethnicity, or religion. Part C (guided response task) required participants to say only positive things about the person (and to continue to inhibit negative responding). Five of the ten items in each part contained a faux pas that tapped ToM ability (e.g., the understanding that Mary would have been frustrated by Noah's 'interruption' because she was studying). In the remaining five items, the intentions of the characters were explicit (e.g., it was made clear verbally and through the emotions displayed that Jack had done the wrong thing to Nadine) and, as such, did not require ToM to make a 'correct' response. Part A was completed first, followed by Part B and Part C. Responses to each item were given a score of 0 if negative, 1 if neutral, and 2 if positive. Total scores for the faux pas and non-faux pas items in each Part were totalled separately (each receiving a score out of 10). Totals for the faux pas and nonfaux pas items were then averaged to calculate total scores out of 10 for each Part. Inhibition difference scores were obtained by subtracting scores on one part from another (B-A, C-A, B-C). Larger inhibition difference scores reflected greater overall change in inhibition response scores from baseline response scores.

Scoring was independently reviewed by two researchers. Differences in opinion of the scoring were discussed and a final score achieved via consensus. Overall there were less than 10 occasions where an item produced an ambiguous response requiring consensus. In all instances, both the content of the utterance and the manner in which it was said were taken into account for scoring (for example, the words 'a nice man' uttered while rolling eyes and with a contradictory tone was taken to be a negative response rather than a positive response).

Standard Neuropsychological Battery. Participants were assessed on standard neuropsychological measures to assist with the validation of the SDT as follows: (1) Disinhibition: Hayling Sentence Completion Test (Burgess \& Shallice, 1997), participants are required to generate semantically related (Part 1; generation) and semantically unrelated (Part 2; inhibition) words to aurally presented sentences. Scaled scores of the overall profile scores were used. (2) Generativity: Controlled Oral Word Association Test (COWAT; Benton, Hamsher, \& Sivan, 1994), requires participants to generate as many words as possible within 1 minute after having been given a letter (C, F, L) to assess letter fluency. Animals fluency (Strauss, Sherman, \& Spreen, 2006), requiring participants to generate the names of as many animals as possible within 1 minute, was also assessed. (3) Processing Speed: the Digit Symbol subtest of the WAIS-III (Wechsler, 1997), requires participants to write down symbols to their corresponding number as fast as possible (scaled scores used), and the Trail Making Test (TMT) Part A (Reitan, 1992), a test which requires participants to connect 25 numbers sequentially in ascending order as quickly as possible. (4) Flexibility: TMT Part B (Reitan, 1992), which required participants to sequentially connect numbers and 
letters in ascending order whilst alternating between the two (i.e., 1-A-2-B-3-C) as fast as possible. (5) Social Cognition: The Awareness of Social Inference-Revised (TASIT-R; McDonald, Flannigan, \& Rollins, 2011) is a measure of social cognition in the form of basic emotion recognition (Part 1), through to the ability to grasp innuendo in the form of basic sarcasm (Part 2) and in more complex forms such as lying (Part 3). The scale scores for each part were used to assess the contribution of social cognition to the task.

Questionnaires tapping social disinhibition: Current Behaviour Scale (CBS). The CBS (Elsass \& Kinsella, 1989) is a 25-item relative-report questionnaire that uses bipolar adjectives rated on a seven-point scale. Higher scores reflect greater disturbance. The scale produces two factor scores, one for Loss of Emotional Control (LEC) and Loss of Motivation (LM). The LEC factor includes items that relate to impulsivity, aggression, and restlessness, whereas the LM factor includes items such as lacking energy, disinterested, and lacking initiative (Kinsella, Packer, \& Olver, 1991). These factors are proposed to reflect disorders of control (LEC) and drive (LM) in individuals with TBI. The CBS had sound psychometric properties, with high internal consistency (Cronbach's $\alpha=.80$ ) and good stability with a correlation of .83 over a 1 -week period (Elsass \& Kinsella, 1989). Factors scale scores were used.

Barratt Impulsivity Scale (BIS-11). The BIS-11 is a 30 item self-report questionnaire, rated on a four-point Likert scale, designed to assess independent constructs of impulsiveness (Patton \& Stanford, 1995). The BIS-11 has a total measure of impulsivity and three second-order factor scores, motor impulsivity, attentional impulsivity, and non-planning, all of which were used in this study. The total and second-order subscale scores from the BIS-11 have shown good test re-test reliability and internal reliability ranging from .59 to .83 (Patton \& Stanford, 1995; Stanford et al., 2009). The BIS-11 has also been validated in a TBI sample (Greve et al., 2001; McHugh, \& Wood, 2008).

Frontal Systems Behavioural Scale (FrSBe). The FrSBe (Grace \& Malloy, 2001) is a 46-item rating scale designed to measure behaviours associated with damage to the fronto-subcortical system brain networks. Only the self-rated disinhibition subscale containing nine items was used for this study. Responses are rated on a five-point Likert scale $(1=$ 'almost never' to $5=$ 'almost always') for current behaviour. Internal consistency is reportedly good for the self-rated forms (Cronbach's $\alpha=.75$ ). The scale has previously been used to de- tect inhibition difficulties in TBI samples (Barrett et al., 2013; O'Keeffe et al., 2007).

Mood Questionnaire: The Depression, Anxiety, and Stress Scale-21 (DASS-21; Lovibond \& Lovibond, 1995) was administered to assess current mood state. The DASS-21 is a 21 -item selfreport measure of the negative emotional states of depression, anxiety, and stress. The measure has acceptable levels of internal reliability: $r=.81$ for the Depression subscale; $r=.73$ for the Anxiety subscale; and $r=.81$ for the Stress subscale (Lovibond \& Lovibond, 1995).

\section{Procedure}

Participants were asked to complete a demographics questionnaire, BIS-11, and FrSBe self-report scale, before being assessed on the SDT, as well as the standard neuropsychological measures. All measures were administered in a single session, with rare exceptions where participants had recently completed (within the last two weeks) a neuropsychological test as a part of another study conducted within the laboratory. Following their participation, TBI participants were given the CBS to give to a close friend of family member who had known them prior to their injury. Informants were provided with a reply paid envelope to mail the questionnaires back to the laboratory. This study was approved by the Human Research Ethics Committee of the University of New South Wales.

\section{Statistical Analyses}

To compare between group performance and latency times across the 3 Parts and 2 Item Types (within subject factors) and to examine the relevant group interactions, a mixed model full information maximum likelihood (FIML) analysis was conducted. The mixed model FIML approach permits a more robust analysis and better accounts for missing data than a traditional general linear model approach (Enders, 2011). An alpha level of $p=.05$ was used to determine significance. Difference scores between parts were calculated to examine overall inhibition performance. $t$-tests were conducted to compare the difference scores across groups. Pearson's correlations were also performed to examine the relationship between overall inhibition performance (Part A - Part B) and standard neuropsychological tests and relative and self-report questionnaires. Correlation sizes only were interpreted according to the guidelines of Cohen (1988). All SDT variables within each group were normally distributed. The assumption of homoscedasticity was violated for the for Part C - Part A inhibition difference score and some standard neuropsychological test comparison 
analyses, and as such, the equal variances not assumed statistic was reported for these.

Alternative mixed models comparison analyses were conducted using years of education entered as a covariate (group differences in education were detected in the subset of participants who completed Part C of the SDT). Additional alternative mixed models comparison analyses were also conducted with DASS-21 scores entered as covariates. Given the inclusion of these variables as covariates did not change the results (i.e., results were consistent with the reported cut-off $p$-value of .05), they were not included in the final analyses. Significant relationships were not detected between time since injury and SDT scores and thus were not controlled for in the analyses $(r=-.100, p=$ .594 to $r=-.205, p=.357$ ).

\section{Results}

\section{Performance on Standard \\ Neuropsychological Tests and Questionnaires}

Descriptive data and comparison statistics for performance in standard neuropsychological tests and questionnaires scores are summarised in Table 1. The TBI group performed significantly worse than the control group on all neuropsychological tests with the exception of the Hayling Sentence Completion task, although a medium effect $(d=.51)$ was present for this test (Cohen, 1988). The TBI participants also displayed higher levels of depression, anxiety, and stress than the control group.

\section{Performance on the SDT}

The FIML mixed models analysis indicated a 2 Group $\times 3$ Part interaction $F(4,241.25)=$ $79.98, p<.001$ that is diagrammatically shown in Figure 1. Post-hoc pairwise comparisons indicated that the participants with TBI responded more positively (i.e., tended to produce positive rather than negative utterances) to items in Part A (the control task) compared to the control participants, $\mathrm{M}_{\text {diff }}=$ $.99, p=.012,95 \% \mathrm{CI}[.22,1.76]$. The reverse pattern was seen in Part B (the inhibition task), i.e., the TBI participants responded less positively to items than the control participants, $\mathrm{M}_{\text {diff }}=1.81$, $p<.001,95 \%$ CI[1.04, 2.58]. No between group differences were observed in Part $\mathrm{C}, \mathrm{M}_{\text {diff }}=.64$, $p=.185,95 \% \mathrm{CI}[-.31,1.59]$.

Additional post-hoc pairwise comparisons for the 2 Group $\times 3$ Part interaction indicated that participants with TBI responded less positively to Part A than both Part B, $\mathrm{M}_{\text {diff }}=1.74, p<.001$, $95 \% \mathrm{CI}[1.16,2.32]$, and Part $\mathrm{C}, \mathrm{M}_{\mathrm{diff}}=3.28, p<$
$.001,95 \% \mathrm{CI}[2.64,3.92]$. They also responded less positively to Part B than Part C, $\mathrm{M}_{\text {diff }}=1.54, p<$ $.001,95 \%$ CI[.90, 2.18]. Similarly, control participants responded less positively to Part A than both Part B, $\mathrm{M}_{\text {diff }}=4.55, p<.001,95 \% \mathrm{CI}[3.86,5.23]$, and Part $\mathrm{C}, \mathrm{M}_{\mathrm{diff}}=4.91, p<.001,95 \% \mathrm{CI}[4.07$, 5.96]. Unlike the TBI participants, there was no difference in responding across Parts $\mathrm{B}$ and $\mathrm{C}$ for the control participants, $\mathrm{M}_{\text {diff }}=.37, p<.001$, $95 \% \mathrm{CI}[-.48,1.21]$.

No overall 2 Group $\times 2$ Item Type interaction was detected $F(2,229.64)=.46, p=.634$. However, there was a significant 2 Group $\times 2$ Item Type $\times 3$ Part interaction, $F(4,229.64)=$ $6.50, p<.001$ that is diagrammatically depicted in Figure 2. Post-hoc group comparison analyses indicated that for Part A (control trial), the TBI participants responded more positively than the control participants on the faux pas items, $\mathrm{M}_{\text {diff }}=1.57$, $p=.003,95 \% \mathrm{CI}[.54,2.53]$. No group differences, however, were detected on the non-faux pas items $\mathrm{M}_{\text {diff }}=.45, p=.378,95 \% \mathrm{CI}[-.55,1.44]$. In Part B (inhibition trial), the TBI participants responded less positively than control participants across both faux pas, $\mathrm{M}_{\text {diff }}=1.93, p<.001,95 \% \mathrm{CI}[.94,2.93]$, and non-faux pas items, $\mathrm{M}_{\mathrm{diff}}=1.69, p=.001$, 95\% CI[.70, 2.69]. In Part C of the SDT (guided response), no group differences were detected across the faux pas, $\mathrm{M}_{\mathrm{diff}}=.76, p=.231,95 \% \mathrm{CI}[-.49$, $2.01]$, and non-faux pas items, $\mathrm{M}_{\mathrm{diff}}=.52, p=$ $.411,95 \% \mathrm{CI}[-.73,1.77]$.

Additional post-hoc analyses for the threeway interaction also indicated that in Part A, both groups responded less positively to the faux pas items than the non-faux pas items (TBI: $\mathrm{M}_{\text {diff }}=$ $1.00, p=.017,95 \%$ CI[.18, 1.82]; control: $\mathrm{M}_{\text {diff }}=$ $2.09, p<.001,95 \% \mathrm{CI}[1.12,3.06])$. In Part B and Part $\mathrm{C}$ both groups responded similarly to the faux pas and non-faux pas tasks (Part B: TBI: $\mathrm{M}_{\text {diff }}=$ $.48, p=.245,95 \% \mathrm{CI}[-.34,1.30]$; control: $\mathrm{M}_{\text {diff }}$ $=.73, p=.142,95 \% \mathrm{CI}[-.24,1.70]$; Part C: TBI: $\mathrm{M}_{\text {diff }}=.26, p=.589,95 \% \mathrm{CI}[-.69,1.21]$; control: $\left.\mathrm{M}_{\mathrm{diff}}=.50, p=.455,95 \% \mathrm{CI}[-.82,1.82]\right)$.

\section{Inhibition Difference Scores}

$t$-test analyses for the inhibition difference scores are shown in Table 2. Consistent with the reported interaction effects noted above, control participants recorded much larger Part B - Part A difference scores than the TBI participants in respect of the total scores and the separate faux pas and non-faux pas scores (i.e., reflecting reduced ability in the TBI participants to inhibit automatic negative responses). Part $\mathrm{C}-$ Part A difference total scores were similar across groups although there was a trend to suggest higher scores for the control 
TABLE 1

Descriptive Statistics of Standard Neuropsychological Tests and Questionnaires by Group with $t$-Test Comparisons

\begin{tabular}{|c|c|c|c|c|c|c|c|}
\hline \multirow[b]{2}{*}{ Test } & \multicolumn{2}{|c|}{ TBI } & \multicolumn{2}{|c|}{ Control } & \multirow[b]{2}{*}{$t$-value } & \multirow[b]{2}{*}{$d$} & \multirow[b]{2}{*}{ Sig } \\
\hline & M & $S D$ & M & $S D$ & & & \\
\hline \multicolumn{8}{|c|}{ Standard neuropsychological measures } \\
\hline Hayling SC SS & 5.00 & 1.76 & 5.73 & 1.03 & $1.46^{\#}$ & .51 & .099 \\
\hline WAIS-III DS SS & 7.57 & 2.94 & 10.80 & 2.54 & 3.50 & 1.18 & .001 \\
\hline TMT - A (time in seconds) & 41.70 & 20.81 & 27.91 & 7.23 & $3.32^{\#}$ & .88 & .002 \\
\hline TMT - B (time in seconds) & 94.34 & 64.95 & 61.95 & 20.16 & $2.52^{\#}$ & .67 & .017 \\
\hline COWAT (CFL) & 33.36 & 9.54 & 44.40 & 11.27 & 3.31 & 1.06 & .002 \\
\hline Animals & 17.40 & 3.83 & 22.60 & 4.69 & 3.82 & 1.21 & $<.001$ \\
\hline \multicolumn{8}{|l|}{ Social cognition: TASIT\# } \\
\hline Part 1 (EET) & 21.31 & 4.80 & 24.60 & 2.06 & $3.28^{\#}$ & .89 & .002 \\
\hline Part 2 (SIM) & 48.14 & 8.00 & 55.63 & 4.37 & $4.13^{\#}$ & 1.16 & $<.001$ \\
\hline Part 3 (SIE) & 50.68 & 8.55 & 55.28 & 4.70 & $2.35^{\#}$ & 1.07 & .024 \\
\hline \multicolumn{8}{|l|}{ Questionnaires } \\
\hline CBS - LEC & 4.85 & 1.18 & - & - & & & \\
\hline CBS - LOM & 3.29 & 0.93 & - & - & & & \\
\hline FrSBe - Disinhibition & 32.33 & 8.01 & - & - & & & \\
\hline \multicolumn{8}{|l|}{ DASS } \\
\hline Depression & 8.64 & 9.83 & 3.20 & 4.79 & $2.64^{\#}$ & .70 & .011 \\
\hline Anxiety & 6.06 & 6.38 & 1.60 & 3.35 & $3.26^{\#}$ & .88 & .002 \\
\hline Stress & 12.00 & 8.81 & 6.50 & 9.31 & 2.13 & .61 & .038 \\
\hline
\end{tabular}

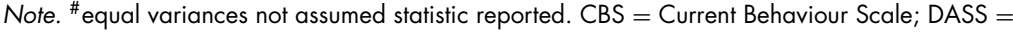
Depression, Anxiety and Stress Scale; FrSBe = Frontal Systems Behavioural Scale; LEC = Loss of Emotional Control; LOM = Loss of Motivation; SC = Sentence Completion; SIE = Social Inference Enriched; SIM = Social Inference Minimal; TASIT = The Awareness of Social Inference Test; TMT = Trail Making Test; SS = Scaled Score.

participants. Part C - Part A difference scores for the control participants were higher than the participants with TBI for the faux pas items but not the non-faux pas items. No group differences were apparent in Part C - Part B difference scores.

\section{Latency Times on the SDT}

The FIML mixed models analysis indicated a 2 Group $\times 3$ Part interaction $F(4,232.43)=5.35$, $p<.001$ for latency times. This interaction is diagrammatically shown in Figure 3. Post-hoc pairwise comparisons indicated that the participants with TBI were quicker in Part A than Part B $\left(\mathrm{M}_{\text {diff }}\right.$ $=1.58, p=.001,95 \% \mathrm{CI}[.66,2.51])$ and Part $\mathrm{C}$ $\left(\mathrm{M}_{\text {diff }}=1.23, p=.001,95 \%\right.$ CI[.20, 2.27] $)$. Control participants were also quicker in Part $\mathrm{A}$ than Part B $\left(\mathrm{M}_{\mathrm{diff}}=1.69, p=.003,95 \% \mathrm{CI}[.59,2.78]\right)$, although no difference between Part A and Part C was detected. Additional post-hoc pairwise comparisons indicated no between group differences in latency for each Part (all $p$ 's $>.05$ ).
A 2 Group $\times 2$ Item Type interaction was also detected $F(2,228.89)=8.45, p<.001$. Post-hoc pairwise comparisons indicated participant latency times were quicker for the faux pas Items than the non-faux pas Items for both the TBI $\left(\mathrm{M}_{\text {diff }}\right.$ $=1.25, p=.002,95 \% \mathrm{CI}[.45,2.05])$ and control participants $\left(\mathrm{M}_{\mathrm{diff}}=1.40, p=.001,95 \% \mathrm{CI}[.39\right.$, 2.41]). Additional post-hoc pairwise comparisons indicated no between group differences in latency times according in each Item Type (all $p$ 's > .05). The mixed models analysis also indicated that no 2 Group $\times 2$ Item Type $\times 3$ Part interaction, $F(4$, $228.89)=.852, p=.494$ ), or group main effect, $F(1,54.11)=1.90, p=.173$, was present.

\section{Correlations Between SDT Inhibition Scores and Other Measures}

Correlations between total inhibition scores (Part A - Part B) and standard neuropsychological tests, social cognition tests, and questionnaires for the overall and TBI samples are shown in Table 3. As 


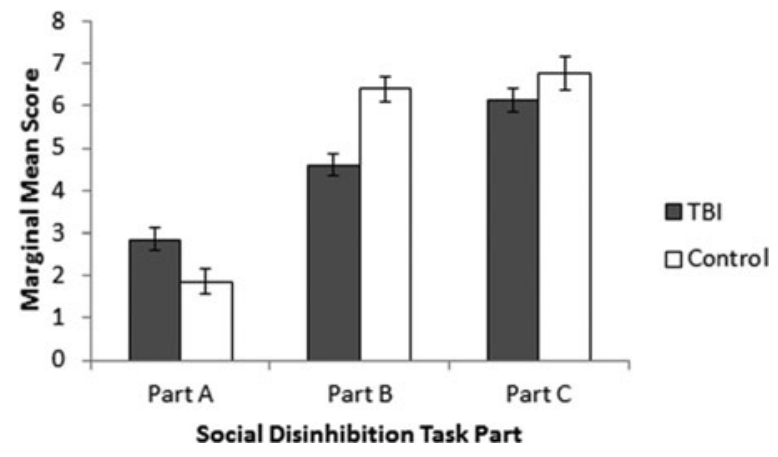

\section{FIGURE 1}

Marginal mean scores for social disinhibition parts stratified by TBI and control groups. Higher values represent more positive responses.

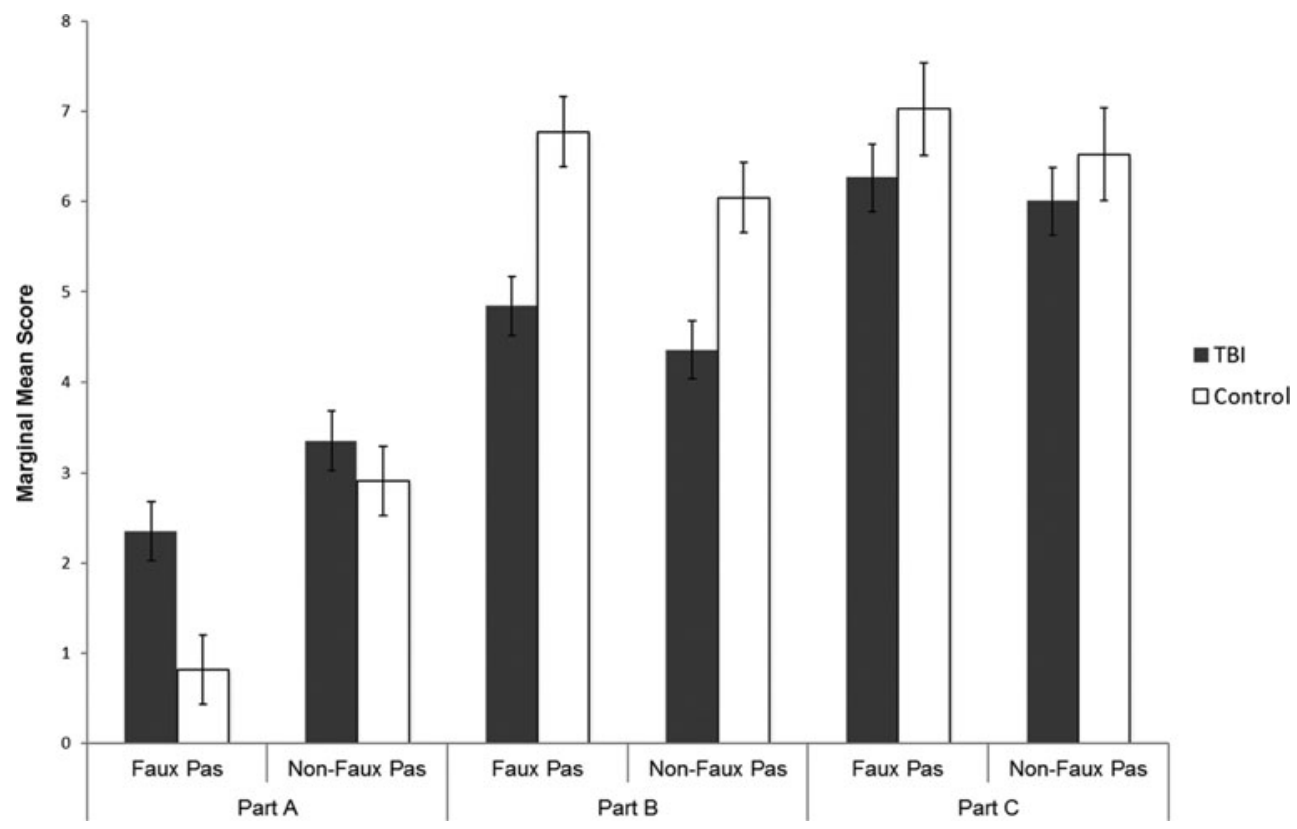

\section{FIGURE 2}

Marginal mean scores for social disinhibition parts stratified by item type (faux pas vs. non-faux pas) and groups (TBI and control). Higher values represent more positive responses.

predicted, the total inhibition score had a moderate to strong positive correlation with the Hayling Sentence Completion Task as well as the TMT A and $\mathrm{B}$ and the COWAT. Small correlations only were detected with the WAIS-IV Digit Symbol and Animals test. The two subset Inhibition scores: faux pas and non-faux pas demonstrated a similar pattern of correlations, although generally somewhat smaller in magnitude. An exception to this was the negligible correlations found between faux pas and TMT scores for the TBI sample. Moderate-tolarge correlations were present between all SDT inhibition scores and TASIT scores. Correlations between the total inhibition scores and the questionnaires were small for the informant-rated CBSLEC (negative correlation) and the CBS-LOM 
TABLE 2

Inhibition Difference Scores Stratified by Group

\begin{tabular}{|c|c|c|c|c|c|c|c|}
\hline \multirow[b]{2}{*}{ Inhibition difference scores } & \multicolumn{2}{|c|}{ TBI } & \multicolumn{2}{|c|}{ Control } & \multirow[b]{2}{*}{ t-value } & \multirow[b]{2}{*}{$d$} & \multirow[b]{2}{*}{ Sig } \\
\hline & $M$ & $S D$ & $M$ & $S D$ & & & \\
\hline \multicolumn{8}{|l|}{ Total scores } \\
\hline Part B - Part A & 3.48 & 4.07 & 9.09 & 3.77 & 5.09 & 1.43 & $<.001$ \\
\hline Part C - Part $A^{\#}$ & 6.57 & 4.87 & 9.17 & 2.98 & 1.96 & 0.64 & .059 \\
\hline Part C - Part B & 2.70 & 4.76 & 1.50 & 2.54 & .81 & 0.31 & .340 \\
\hline \multicolumn{8}{|l|}{ Faux Pas } \\
\hline Part B - Part A & 2.48 & 2.63 & 5.95 & 2.03 & 5.18 & 1.48 & $<.001$ \\
\hline Part C - Part $A^{\#}$ & 3.78 & 2.65 & 6.00 & 1.04 & 3.53 & 1.10 & .001 \\
\hline Part C - Part B & 1.09 & 2.61 & 0.67 & 1.72 & 0.50 & 0.19 & .619 \\
\hline \multicolumn{8}{|l|}{ Non-faux pas } \\
\hline Part B - Part A & 1.00 & 2.57 & 3.14 & 2.23 & 3.18 & 0.89 & .003 \\
\hline Part C - Part A & 2.78 & 3.16 & 3.17 & 2.59 & 0.36 & 0.13 & .720 \\
\hline Part C - Part B & 1.61 & 2.71 & 0.83 & 1.70 & 0.90 & 0.34 & .375 \\
\hline
\end{tabular}

Note. "Equal variances not assumed statistic reported.

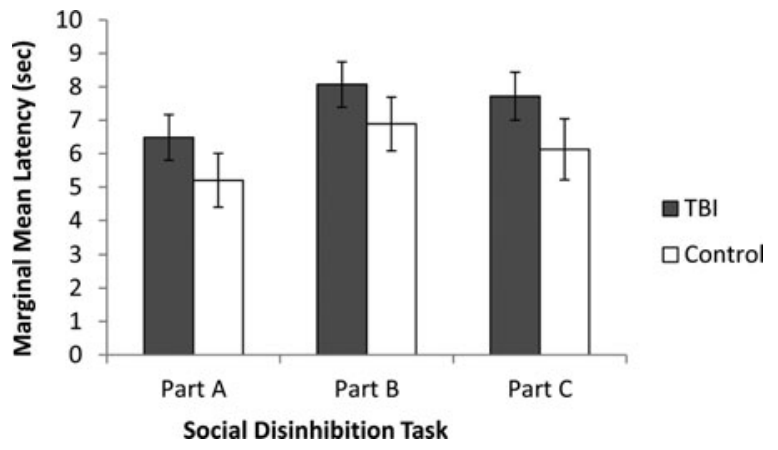

\section{FIGURE 3}

Marginal mean latency times in seconds for social disinhibition parts stratified by item type (faux pas vs. non-faux pas) and groups (TBI and control).

(positive) and small to negligible for the FrSBe - Disinhibition and BIS-11.

\section{Discussion}

This study aimed to pilot the SDT as a potential new clinical measure of social disinhibition for individuals with TBI. In doing so, it also sought to determine whether problems of social inhibition are dependent on ToM ability and whether explicit guidance could correct social disinhibitory problems.

In general, performance on the SDT was as expected. When participants were asked to say the first word that came to mind about a particular character in awkward social scenes (control task - Part
A), all participants tended to respond negatively. Interestingly, control participants responded less positively than participants with TBI when these scenes entailed a faux pas which, by necessity, required understanding of implicit mental states. One explanation for this is that, as previously documented, the participants with TBI had difficulty understanding ToM (e.g., Honan et al., 2015; McDonald \& Flanagan, 2004; McDonald et al., 2014; Milders et al., 2006, 2008; Muller et al., 2010) so automatic negative evaluations were triggered to a lesser degree.

When participants were asked to inhibit their automatic negative responses (inhibition task - Part B), the individuals with TBI were less able than controls leading to less positive utterances being 


\section{TABLE 3}

Correlations Between SDT Inhibition Scores and Alternative Measures for Overall and TBI Samples

\begin{tabular}{|c|c|c|c|c|c|c|}
\hline & \multicolumn{2}{|c|}{$\begin{array}{l}\text { Total inhibition } \\
\text { scores }\end{array}$} & \multicolumn{2}{|c|}{$\begin{array}{c}\text { Faux pas } \\
\text { inhibition score }\end{array}$} & \multicolumn{2}{|c|}{$\begin{array}{l}\text { Non-faux pas } \\
\text { inhibition score }\end{array}$} \\
\hline & Overall & $\mathrm{TBI}$ & Overall & $\mathrm{TBI}$ & Overall & $\mathrm{TBI}$ \\
\hline \multicolumn{7}{|l|}{ Standard neuropsychological measures } \\
\hline Haylings SC Total SS ( $n=42,27)$ & .448 & .417 & .352 & .322 & .383 & .325 \\
\hline WAIS-III DS SS (n=38,23) & .238 & .300 & .174 & .233 & .214 & .245 \\
\hline TMT A - Time in seconds $(n=51,24)$ & -.470 & -.249 & -.419 & .051 & -.396 & -.429 \\
\hline TMT B - Time in seconds $(n=49,29)$ & -.448 & -.350 & -.356 & -.062 & -.418 & -.515 \\
\hline COWAT (CFL) $(n=40,25)$ & .369 & .319 & .323 & .188 & .267 & .289 \\
\hline Animals $(n=40,25)$ & .218 & .133 & .246 & .130 & .099 & .073 \\
\hline \multicolumn{7}{|l|}{ Social cognition } \\
\hline TASIT Part 1 (EET) $(n=49,29)$ & .495 & .520 & .423 & .413 & .433 & .422 \\
\hline TASIT Part 2 (SIM) $(n=47,28)$ & .522 & .581 & .476 & .474 & .410 & .448 \\
\hline TASIT Part 3 (SIE) $(n=46,28)$ & .371 & .432 & .268 & .266 & .375 & .425 \\
\hline \multicolumn{7}{|l|}{ Questionnaires } \\
\hline CBS - LEC $(n=23)$ & - & -.174 & - & -.240 & - & -.036 \\
\hline $\operatorname{CBS}-\operatorname{LOM}(n=23)$ & - & .180 & - & .075 & - & .199 \\
\hline FrSBe - Disinhibition $(n=27)$ & - & -.162 & - & -.223 & - & -.027 \\
\hline $\begin{array}{l}\text { Barrett Impulsivity Scale } 11 \\
(n=36,21)\end{array}$ & -.144 & -.052 & -.070 & .033 & -.179 & -.125 \\
\hline
\end{tabular}

Note. Correlations greater than $r=.25$, indicating at least a moderate effect size, are highlighted in bold text (Cohen, 1988). See explanatory note. Overall sample size and TBI sample size, respectively, are shown in brackets. followed by total inhibition scores $=$ Part A - Part B. CBS $=$ Current Behaviour Scale; COWAT $=$ Controlled Oral Word Association Test; DS = Digit Symbol; EET = Emotion FrSBe = Frontal Systems Behaviour Scale; LEC = Loss of Emotional Control; LOM = Loss of Motivation; SIE = Social Inference Enriched; SIM = Social Inference Minimal; SR = Self-report; SS = Scaled Score; TASIT $=$ The Awareness of Social Inference Test; TMT = Trail Making Test.

made. This finding is consistent with prior studies that found similar deficits in social inhibition ability using ecologically valid experimental tasks and observational testing paradigms (Honan et al., 2015; McDonald et al., 2014; Osborne-Crowley et al., 2015). They are also consistent with studies that documented social disinhibition difficulties in TBI using informant- and self-report measures (e.g., Barrett et al., 2013; Monsalve et al., 2012; O'Keeffe, Dockree, Moloney, Carton, \& Robertson, 2007). Interestingly, this failure to inhibit was apparent on both the non-faux pas and faux pas items, despite the fact that the group with TBI appeared to have generally less to inhibit on the latter, given the relatively positive utterances that were made for faux pas items on Part A. Indeed, in general, it appeared that faux pas and non-faux pas items produced a similar pattern of inhibitory performance in both groups. This suggests that the impairment in Part B reflected a general failure to inhibit a socially inappropriate response regardless of how accurately the social situation was appraised or how much appraisal demanded mentalising ability.
An additional aim of the study was to examine whether TBI individuals were able to adjust their responding when provided with specific guidelines about how they should respond. Specifically, Part $\mathrm{C}$ of the SDT asked participants to not only inhibit the automatic negative responses, but also replace these responses with more positive utterances about the character. The results indicated TBI and control participants responded in a similar manner across the faux pas and non-faux pas items. Furthermore, though performance for the control participants was similar across Parts B and C, performance improved for the TBI participants from Part $B$ to $C$ suggesting that this guidance was helpful to them. Importantly, these findings suggest that TBI individuals who are socially disinhibited may benefit from rehabilitation efforts or training in how to respond more appropriately in negative social situations. This fits with other work that has demonstrated how external cues can remediate goal neglect and facilitate behavioural regulation during novel complex task performance (Manly, Hawkins, Evans, Woldt, \& Robertson, 2002). 
Hypotheses concerning the convergent validity of the SDT were generally upheld. Total Inhibition scores were associated with Hayling Sentence Completion task scores, another test purpose designed to measure disinhibition. In addition, SDT scores correlated with other standard neuropsychological tests of executive functions, especially generativity. SDT scores were also generally associated with cognitive flexibility. A specific exception to this was the negligible correlation detected in the TBI sample between faux pas inhibition scores and cognitive flexibility, indicating that there may be minimal involvement of cognitive flexibility skills when inhibiting responses to information requiring higher level social processing (i.e., ToM). The social dimension of the SDT was further supported by its strong association with TASIT a measure of social cognition.

Ecological validity was less clearly determined. The small associations found with selfreport measures of disinhibition are consistent with prior studies indicating reduced self-awareness of inhibition ability in TBI individuals (O'Keeffe et al., 2007). Unexpectedly, small associations were also found with our informant-reported measure, the CBS-LEC. This may be due to the fact that the items in the CBS-LEC were more reflective of the ability to control emotions rather than the ability to control verbal social responses. It is also likely that the SDT, like other laboratory based tasks, has limited ability to predict general behaviour in everyday settings. Correlation with a more proximal measure of actual social behaviour, such as in vivo observation (OsborneCrowley et al., 2015) may be a better means to demonstrate ecological validity.

Overall, these results are promising for the SDT as a new clinical tool to measure social disinhibition. It offers an advance over standard clinical tests of response inhibition and interference control that are often used to infer social inhibition difficulties in TBI. Such tests are problematic as they do not contain items that are imbedded in social context and therefore lack ecological validity. Furthermore, such measures partly reflect processing speed. For example, scores on the Hayling Sentence Completion Test (Burgess \& Shallice, 1997) are derived by summing inhibition performance scores and reaction times across both control and inhibition task trials. Time limits (or time taken to complete) are also imposed in most Stroop tasks (e.g., Stroop Neuropsychological Screening Test; Trenerry, 1989) and thus performance is reflective of underlying processing speed. It is of interest to note that the Stroop task was found in one meta-analysis study (Dimoska-Di Marco, McDonald, Kelly, Tate, \& Johnstone, 2011) to lack utility in detecting interference control difficulties in individuals with TBI as notable effect sizes were only present in studies using 'total time' taken to complete the task as the outcome variable. This was argued to be due to factors such as response speed, fatigue, and arousal rather than inhibitory control per se.

In contrast, SDT scores were not influenced by response time. In particular, though response latency times were predictably slower in the inhibition task (Part B) than the control task (Part A) for both TBI and control participants, and response latency times for the control participants were slower in the guided response task (Part C) than the control task, no between group differences were detected. A small association was also only present between overall inhibition performance and processing speed (as measured by the WAIS-III Digit Symbol subtest). Response time was not, therefore, a significant mediator of social inhibition ability as assessed by the SDT.

Despite these promising results, it is important to note that the SDT is limited as a decontextualised test of social performance. An assumption of this study and the development of the SDT is that more positive responses when presented with negative social information are akin to appropriate social responding. However, different social environments provide different contextual constraints and the ability to regulate automatic responses needs to be reflexive to such nuances (Hooker \& Knight, 2006). What is considered socially acceptable while at home with a partner may be very different to what is considered socially acceptable at a formal black-tie event or business meeting (i.e., where there is high expectation to contain inappropriate behaviour). It may also be the case that excessively positive comments may be socially inappropriate if, for example, they cause another person embarrassment. Future research in this area may help delineate the extent to which varying social contexts impact on social inhibition ability in TBI individuals.

Additional limitations of the study need to be noted. First, responses to the social information were subjectively scored as negative, neutral, or positive. Although scoring was reviewed by a second researcher, this process was not blind. This process was adopted as part of the development of the instrument including the development of a scoring protocol that could encompass the pragmatic dimension of responses (e.g., saying 'a nice man' while rolling eyes and with a contradictory tone). Future validation should examine the reliability of blinded scoring, e.g., via videotaping responses. Secondly, items in SDT were not counterbalanced across parts. As such, we are unable to rule out 
any possible effects of order. However, given the content of the items were qualitatively balanced across the three Parts, any possible order effects are expected to be negligible. Third, the majority of participants in this study were male. This is a direct reflection of the high proportion of TBI participants, in which high rates of incidence in males (3:1 male to female ratio in severe TBI) are frequently reported (Tate, McDonald, \& Lulham, 1998). It would be pertinent to examine possible gender differences on the SDT in future studies. Fourth, given general intellectual ability was not measured in this study, the extent to which SDT scores are influenced by general intellectual ability is not known and thus should be explored in future research. Finally, the extent to which 'inhibition performance' may reflect verbal comprehension difficulties was not examined in this study. However, given individuals with TBI were able to perform at levels similar to control participants in Part A (non-faux pas) items and across all Part C items, and given change was present between Part A and Part B scores in the TBI participants (i.e., indicating response adjustment took place), it is unlikely that comprehension difficulties contributed to the present results.

In conclusion, difficulties in social disinhibition are a common consequence of TBI. This study has detailed an examination of a new measure of social disinhibition, the SDT. The SDT discriminated people with TBI from controls and demonstrated convergent validity with another measure of disinhibition, other tests of executive function and a test of social cognition. Importantly, the SDT did not seem to be reliant on processing speed, unlike other standard measures of inhibition. Overall, the SDT appears to be suitable to detect social inhibition difficulties in a clinical setting. The test also indicated improved responding with guidance, providing a new direction for remediation of social inhibition, e.g., by the use of cue detection training. Future studies are needed to confirm that rater scoring is reliable for the SDT and also, to further examine the ecological validity of the test.

\section{Acknowledgement}

We are particularly grateful to the people with traumatic brain injuries and our control participants who gave willingly of their time to assist this research.

\section{Funding}

This research was funded by a University of New South Wales Science Faculty Early Career Research Grant.

\section{Conflict of Interest}

None.

\section{Ethical Standards}

The authors assert that all procedures contributing to this work comply with the ethical standards of the relevant national and institutional committees on human experimentation and with the Helsinki Declaration of 1975, as revised in 2008.

\section{References}

Arciniegas, D.B., \& Wortzel, H.S. (2014). Emotional and behavioral dyscontrol after traumatic brain injury. Psychiatric Clinics of North America, 37(1), 31-53.

Barrett, R.D., McLellan, T.L., \& McKinlay, A. (2013). Self versus family ratings of the frontal systems behaviour scale and measured executive functions: Adult outcomes following childhood traumatic brain injury. PLoS One, 8(10), e76916.

Beer, J.S., John, O.P., Scabini, D., \& Knight, R.T. (2006). Orbitofrontal cortex and social behavior: Integrating self-monitoring and emotion-cognition interactions. Journal of Cognitive Neuroscience, 18(6), 871-879.

Benton, A.L., Hamsher, K.D., \& Sivan, A.B. (1994). Multilingual aphasia examination (3rd ed.). San Antonio, TX: Psychological Corporation.

Bibby, H., \& McDonald, S. (2005). Theory of mind after traumatic brain injury. Neuropsychologia, 43(1), 99114.

Brownell, H., \& Martino, G. (1988). Deficits in inference and social cognition: The effects of right hemisphere brain damage on discourse. In M. Breman \& C. Chiarello (Eds.), Right hemisphere language comprehension: Perspectives from cognitive neuroscience (pp. 309-328). Mahwah, NJ: Lawrence Erlbaum Associates.

Burgess, P.W., \& Shallice, T. (1997). The hayling and brixton tests. Thurston, Suffolk: Thames Valley Test Company.

Ciurli, P., Formisano, R., Bivona, U., Cantagallo, A., \& Angelelli, P. (2011). Neuropsychiatric disorders in persons with severe traumatic brain injury: Prevalence, phenomenology, and relationship with demographic, clinical, and functional features. The Journal of Head Trauma Rehabilitation, 26(2), 116-126.

Cohen, J. (1988). Statistical power analysis for the behavioral sciences (2nd ed.) Hillsdale, New Jersey: L Erlbaum.

Conners, C.K., \& Staff, M. (2000). Conners' continuous performance test II (CPT II V. 5). North Tonawanda, NY: Multi-Health Systems Inc.

Dimoska-Di Marco, A., McDonald, S., Kelly, M., Tate, R., \& Johnstone, S. (2011). A meta-analysis of response inhibition and Stroop interference control deficits in adults with traumatic brain injury (TBI). Journal of Clinical and Experimental Neuropsychology, 33(4), 471-485. 
Draper, K., \& Ponsford, J. (2008). Cognitive functioning ten years following traumatic brain injury and rehabilitation. Neuropsychology, 22(5), 618.

Elsass, L., \& Kinsella, G. (1989). Development of a scale for measuring behaviour change following closed head injury. Paper presented at the Proceedings of the Fourteenth Annual Brain Impairment Conference.

Enders, C.K. (2011). Analyzing longitudinal data with missing values. Rehabilitation Psychology, 56(4), 267.

Grace, J., \& Malloy, P.F. (2001). FrSe, frontal systems behavior scale: Professional manual. Lutz, FL: Psychological Assessment Resources, Inc.

Greve, K.W., Sherwin, E., Stanford, M.S., Mathias, C., Love, J., \& Ramzinski, P. (2001). Personality and neurocognitive correlates of impulsive aggression in long-term survivors of severe traumatic brain injury. Brain Injury, 15, 255-262.

Honan, C.A., McDonald, S., Gowland, A., Fisher, A., \& Randall, R.K. (2015). Deficits in comprehension of speech acts after TBI: The role of theory of mind and executive function. Brain and Language, 150, 69-79.

Honan, C.A., McDonald, S., Sufani, C., Hine, D.W., \& Kumfor, F. (2016). The awareness of social inference test: Development of a shortened version for use in adults with acquired brain injury. The Clinical Neuropsychologist, 30(2), 243-264.

Hooker, C.I., \& Knight, R.T. (2006). The role of lateral orobitofrontal cortex in the inhibitory control of emotion. In D.H. Zald \& S.L. Rauch (Eds.), The Orbitofrontal Cortex (pp. 307-423). Oxford, United Kingdom: Oxford University Press.

Kilmer, R.P., Demakis, G.J., Hammond, F.M., Grattan, K.E., Cook, J.R., \& Kornev, A.A. (2006). Use of the neuropsychiatric inventory in traumatic brain injury: A pilot investigation. Rehabilitation Psychology, 51(3), 232.

Kinsella, G., Packer, S., \& Olver, J. (1991). Maternal reporting of behaviour following very severe blunt head injury. Journal of Neurology, Neurosurgery \& Psychiatry, 54(5), 422-426.

Knutson, K.M., Dal Monte, O., Schintu, S., Wassermann, E. M., Raymont, V., Grafman, J., \& Krueger, F. (2015). Areas of brain damage underlying increased reports of behavioral disinhibition. The Journal of Neuropsychiatry and Clinical Neurosciences, 27, 193-198.

Lee, T.M., Ip, A.K., Wang, K., Xi, C.-H., Hu, P.-P., Mak, H.K., . . Chan, C.C. (2010). Faux pas deficits in people with medial frontal lesions as related to impaired understanding of a speaker's mental state. Neuropsychologia, 48(6), 1670-1676.

Lovibond, P.F., \& Lovibond, S.H. (1995). Manual for the depression anxiety stress scales. Sydney, Australia: Psychology Foundation of Australia. Sydney, Australia: Psychology Foundation of Australia.

Løvstad, M., Funderud, I., Endestad, T., Due-Tønnessen, P., Meling, T., Lindgren, M.,. . . Solbakk, A.-K.
(2012). Executive functions after orbital or lateral prefrontal lesions: Neuropsychological profiles and self-reported executive functions in everyday living. Brain Injury, 26(13-14), 1586-1598.

Malloy, P., Bihrle, A., Duffy, J., \& Cimino, C. (1993). The orbitomedial frontal syndrome. Archives of Clinical Neuropsychology, 8(3), 185-201.

Manly, T., Hawkins, K., Evans, J., Woldt, K., \& Robertson, I.H. (2002). Rehabilitation of executive function: Facilitation of effective goal management on complex tasks using periodic auditory alerts. $\mathrm{Neu}$ ropsychologia, 40(3), 271-281.

Marosszeky, N.E.V. (1998). The PTA Protocol: Guidelines for using the Westmead Post-Traumatic Amnesia (PTA) Scale. Department of Rehabilitation Medicine, Westmead Hospital and Department of Psychology, Macquarie University, Wild \& Woolley.

McDonald, S., \& Flanagan, S. (2004). Social perception deficits after traumatic brain injury: interaction between emotion recognition, mentalizing ability, and social communication. Neuropsychology, 18(3), 572-579. doi:10.1037/0894-4105.18.3.572

McDonald, S., Flanagan, S., \& Rollins, J. (2011). The Awareness of Social Inference Test (Revised). Sydney, Australia: Pearson Assessment.

McDonald, S., Gowland, A., Randall, R., Fisher, A., Osborne-Crowley, K., \& Honan, C. (2014). Cognitive factors underpinning poor expressive communication skills after traumatic brain injury: Theory of mind or executive function?. Neuropsychology, 28(5), 801.

McDonald, S., Honan, C., Kelly, M., Byom, L., \& Rushby, J. (2013). Disorders of social cognition and social behaviour following severe TBI. In S. McDonald, L. Togher, \& C. Code (Eds.) Social and communication disorders following traumatic brain injury (2nd ed., pp. 119-159). Hove, UK: Psychology Press.

McHugh, L., \& Wood, R. L. (2008). Using a temporal discounting paradigm to measure decision-making and impulsivity following traumatic brain injury: a pilot study. Brain Injury, 22, 715-721.

Milders, M., Ietswaart, M., Crawford, J. R., \& Currie, D. (2008). Social behavior following traumatic brain injury and its association with emotion recognition, understanding of intentions, and cognitive flexibility. Journal of the International Neuropsychological Society, 14, 318-326.

Milders, M., Ietswaart, M., Currie, D., \& Crawford, J.R. (2006). Impairments in theory of mind shortly after traumatic brain injury and ar 1-year follow-up. Neuropsychology, 20, 400-408.

Monsalve, B.C., Guitart, M.B., Lopez, R., Vilasar, A.B., \& Quemada, J.I. (2012). Psychopathological evaluation of traumatic brain injury patients with the Neuropsychiatric Inventory. Revista de Psiquiatría y Salud Mental (English Edition), 5(3), 160-166.

Muller, F., Simion, A., Reviriego, E., Galera, C., Mazaux, J.-M., Barat, M., \& Joseph, P.-A. (2010). Exploring 
theory of mind after severe traumatic brain injury. Cortex, 46, 1088-1099.

Nigg, J.T. (2000). On inhibition/disinhibition in developmental psychopathology: Views from cognitive and personality psychology and a working inhibition taxonomy. Psychological Bulletin, 126(2), 220.

O'Keeffe, F., Dockree, P., Moloney, P., Carton, S., \& Robertson, I. (2007). Characterising error-awareness of attentional lapses and inhibitory control failures in patients with traumatic brain injury. Experimental Brain Research, 180(1), 59-67.

Ochsner, K.N., \& Lieberman, M.D. (2001). The emergence of social cognitive neuroscience. American Psychologist, 56(9), 717-734. doi:10.1037/0003066X.56.9.717.

O'Keeffe, F., Dockree, P., Moloney, P., Carton, S., \& Robertson, I.H. (2007). Awareness of deficits in traumatic brain injury: A multidimensional approach to assessing metacognitive knowledge and onlineawareness. Journal of the International Neuropsychological Society, 13(01), 38-49.

Osborne-Crowley, K., McDonald, S., \& Francis, H. (2015). Development of an observational measure of social disinhibition after traumatic brain injury. Journal of Clinical and Experimental Neuropsychology, 38, 341-353.

Patton, J. H., \& Stanford, M. S. (1995). Factor structure of the Barratt Impulsiveness Scale. Journal of Clinical Psychology, 51, 768-774

Premack, D., \& Woodruff, G. (1978). Does the chimpanzee have a theory of mind? Behavioral and Brain Sciences, 1(04), 515-526. doi:10.1017/S0140525X00076512

Reitan, R. M. (1992). Trail Making Test. Tucson, AZ: Reitan Neuropsychological Laboratories.

Rolls, E.T., Hornak, J., Wade, D., \& McGrath, J. (1994). Emotion-related learning in patients with social and emotional changes associated with frontal lobe dam- age. Journal of Neurology, Neurosurgery \& Psychiatry, 57(12), 1518-1524.

Russell, W.R., \& Smith, A. (1961). Post-traumatic amnesia in closed head injury. Archives of Neurology, 5(1), 4-17.

Senathi-Raja, D., Ponsford, J., \& Schönberger, M. (2010). Impact of age on long-term cognitive function after traumatic brain injury. Neuropsychology, 24(3), 336

Stanford, M. S., Mathias, C. W., Dougherty, D. M., Lake, S. L., Anderson, N. E., \& Patton, J. H. (2009) Fifty years of the Barratt Impulsiveness Scale: An update and review. Personality and Individual Differences, 47, 385-395.

Strauss, E., Sherman, E.M.S., \& Spreen, O. (2006). A compendium of neuropsychological tests. New York: Oxford University Press.

Tate, R., McDonald, S., \& Lulham, J. (1998). Incidence of hospital-treated traumatic brain injury in an Australian community. Australian and New Zealand Journal of Public Health, 22, 419-423.

Tate, R.L., Broe, G.A., Cameron, I.D., Hodgkinson, A.E., \& Soo, C.A. (2005). Pre-injury, injury and early post-injury predictors of long-term functional and psychosocial recovery after severe traumatic brain injury. Brain Impairment, 6(02), 75-89.

Teasdale, G.M. (1995). Head injury. Journal of Neurology, Neurosurgery \& Psychiatry, 58(5), 526.

Trenerry, M.R. (1989). Stroop neuropsychological screening test: manual: Psychological Assessment Resources.

Wechsler, D. (1997). Wechsler adult intelligence scaleThird Edition (WAIS-III). San Antonio, TX: Psychological Corporation.

Wood, R.L., \& Rutterford, N.A. (2006). Demographic and cognitive predictors of long-term psychosocial outcome following traumatic brain injury. Journal of the International Neuropsychological Society, 12(03), 350-358. 